\title{
Connecting sympathetic and renin-angiotensin system overdrive in neurogenic hypertension through miRNA-181a
}

\author{
Vinicia C. Biancardi ${ }^{1,2} \cdot$ Neeru M. Sharma ${ }^{3}$ \\ Received: 20 May 2020 / Revised: 23 May 2020 / Accepted: 23 May 2020 / Published online: 18 June 2020 \\ (c) The Japanese Society of Hypertension 2020
}

Hypertension is a primary risk factor for cardiovascular and renal impairments that increases the likelihood of heart disease and stroke, two leading causes of mortality in the United States. The pathogenesis of hypertension is complex and multifactorial. Substantial experimental and patient data suggest that the combined effects of an overactivated sympathetic nervous system (SNS) and renin-angiotensin system (RAS) lead to the initiation and maintenance of hypertension. However, the underlying regulatory mechanisms of aberrant SNS and RAS in hypertension, particularly those determined by a genetic predisposition that may link both abnormalities, remain mostly elusive. Circulating and tissue-specific microRNAs (miRNAs) have emerged as potent regulators of various cellular processes. Abnormalities in the expression of these miRNAs can result in pathological conditions, including hypertension [1, 2]. miRNAs are a group of highly conserved, short noncoding RNAs (20-23 nucleotides long) that posttranscriptionally regulate gene expression through a partial base-pairing mechanism, which results in translational inhibition and/or mRNA degradation [3]. The interaction between the miRNA "seed" sequence (positions 2-8) and the complementary "seed match" sequence in the $3^{\prime}$ untranslated regions (UTRs) of mRNA determines the miRNA targets [4]. This low complementarity requirement between miRNA and its $3^{\prime}$ UTR target mRNA enables a single miRNA to modulate many pathways and processes by simultaneously targeting multiple proteins [5].

Neeru M. Sharma

nsharma@unmc.edu

1 Anatomy, Physiology, \& Pharmacology, College of Veterinary Medicine, Auburn University, Auburn, AL, USA

2 Center for Neurosciences Research Initiative, Auburn University, Auburn, AL, USA

3 Department of Cellular and Integrative Physiology, University of Nebraska Medical Center, Omaha, NE, USA
In the current issue of Hypertension Research, the data presented by Jackson et al. suggest a causative role for miR181a in blood pressure regulation. Employing a mouse model of hypertension (the Schlager hypertensive mouse$\mathrm{BPH} / 2 \mathrm{~J}$ ), the findings point to a prohypertensive interplay between renal sympathetic nerve activity and miR-181amediated RAS activity [6]. This is particularly interesting from a translational perspective, considering previous work from Brian Morris' and Geoff Head's laboratories demonstrating differential miR-181a expression in hypertensive human kidneys, implicating miR-181a in renin mRNA regulation [7], and illustrating an inverse relationship between miR-181a and renin in blood pressure elevation in $\mathrm{BPH} / 2$ mice [8]. While their previous work showed an association between sympathetic overactivation in the BPH/ 2 mouse and miR-181a-mediated RAS regulation (reviewed in [9]), the extent to which miR-181a might act as a causal missing link between SNS and RAS crosstalk was unknown.

In the present study, the authors employed intravenous injections of renal-targeted miR-181a mimic, telemetry blood pressure recordings, and pharmacological assessment of RAS in BPH/2J mice. Treated mice exhibited transient decreases in arterial blood pressure, reduced renal renin mRNA expression, and lacked a depressive response to angiotensin-converting enzyme inhibition. Furthermore, the authors found that bilateral renal denervation increased the expression of miR-181a and its targeting transcription factor, $T c f 7 l 2$, with a concurrent reduction in renal renin mRNA expression, reversing hypertension in $\mathrm{BPH} / 2 \mathrm{~J}$ mice. Altogether, their findings suggest that renal sympathetic nerves play a role in downregulating renal miR-181a, which appears to be a novel contributor to RAS overactivity by modulating renin levels in vivo and thereby elevating BP in hypertensive BPH/2J mice (Fig. 1).

As acknowledged by the authors, many questions remain to be elucidated. While the data support a link between the differential abundance of miR-181a and hypertension due to high sympathetic activity, it is unclear how renal 


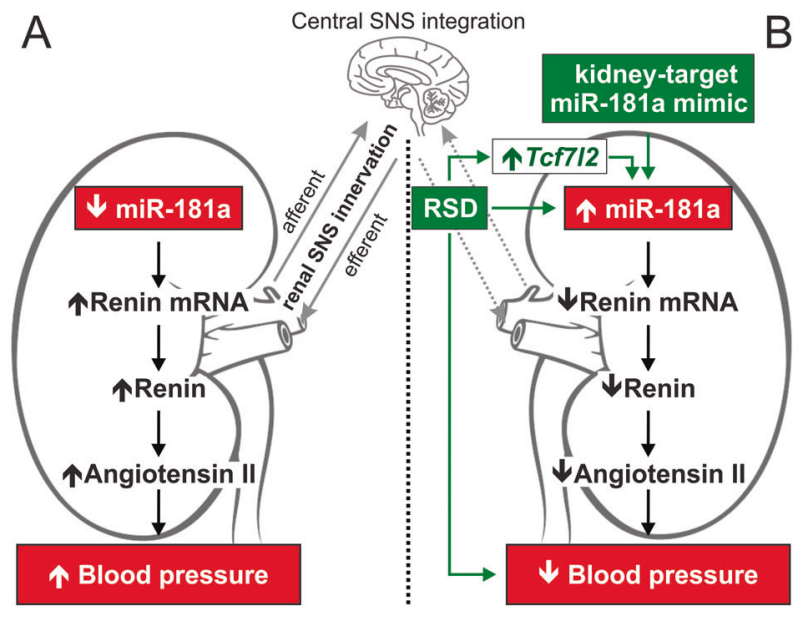

Fig. 1 The miRNA-181a-mediated mechanism in regulating sympathetic and renin-angiotensin-system overdrive in neurogenic hypertension. (a) miR-181a is downregulated in Schlager genetically hypertensive mice $(\mathrm{BPH} / 2)$, which negatively regulates renin mRNA expression, leading to increased blood pressure. Concurrently, BPH/2J mice display sympathetic hyperinnervation of the kidney, contributing to increased SNS activity in this model. (b) The in vivo administration of renal-targeted miR181-a mimic decreases renal renin mRNA and the depressor response to angiotensin-converting enzyme inhibition, suggesting a role of miR-181a in the regulation of RAS during hypertension. Furthermore, renal denervation (RSD) is associated with increased $T c f 7 l 2 \mathrm{mRNA}$ levels leading to higher miR-181a abundance in BPH/2J mice, presenting a possible mechanism by which SNS and RAS crosstalk is linked in this model of neurogenic hypertension

denervation normalizes the expression of $T c f 7 l 2$ to allow for increased miRNA-181a levels. Furthermore, although the miR-181a mimic was specifically targeted to the kidney, it is not entirely clear which renal cell types express this miRNA. While the authors speculate that the effects of miR-181a are primarily on intrarenal (tubular) renin expression rather than circulating renin levels, the mechanism(s) underlying the ability of tubular renin to regulate blood pressure independently of juxtaglomerular cell-derived circulating renin require further investigation. In addition, it would be of interest to examine whether the mechanism proposed by Jackson et al. is present in other models of hypertension and cardiovascular diseases associated with SNS and RAS abnormalities, as well as the potential involvement of other miRNAs. For instance, our group [10] has recently shown that miR-133a contributes to the regulation of angiotensinogen within the central nervous system, leading to increased SNS activity during heart failure. Of interest, previous work shows that decreased blood pressure in patients with hypertension who have undergone renal denervation is associated with increased serum levels of miR-133a over time. However, whether the changes in miR-133a expression were directly related to the reduction in SNS activity, decreased arterial blood pressure itself, or a combination thereof was not determined [11]. Nevertheless, this work illustrates the importance of miRNAs in cardiovascular pathologies, emphasizing the complexities of arterial blood pressure regulation in the hypertensive state, and highlighting the potential for miRNAs to serve as biomarkers for treatment efficacy and endorgan damage.

\section{Compliance with ethical standards}

Conflict of interest The authors declare that they have no conflict of interest.

Publisher's note Springer Nature remains neutral with regard to jurisdictional claims in published maps and institutional affiliations.

\section{References}

1. Jusic A, Devaux Y, Action EU-CC. Noncoding RNAs in hypertension. Hypertension. 2019;74:477-92.

2. van Rooij E. The art of microRNA research. Circulation Res. 2011;108:219-34.

3. Bartel DP. MicroRNAs: genomics, biogenesis, mechanism, and function. Cell. 2004;116:281-97.

4. Lewis BP, Burge CB, Bartel DP. Conserved seed pairing, often flanked by adenosines, indicates that thousands of human genes are microRNA targets. Cell. 2005;120:15-20.

5. Mallanna SK, Rizzino A. Emerging roles of microRNAs in the control of embryonic stem cells and the generation of induced pluripotent stem cells. Dev Biol. 2010;344:16-25.

6. Jackson KL, Gueguen C, Lim K, Eikelis N, Stevenson ER, Charchar FJ, et al. Neural suppression of miRNA-181a in the kidney elevates renin expression and exacerbates hypertension in Schlager mice. Hypertens Res. 2020. https://doi.org/10.1038/ s41440-020-0453-x.

7. Marques FZ, Campain AE, Tomaszewski M, ZukowskaSzczechowska E, Yang YH, Charchar FJ, et al. Gene expression profiling reveals renin mRNA overexpression in human hypertensive kidneys and a role for microRNAs. Hypertension. 2011;58:1093-8.

8. Jackson KL, Marques FZ, Watson AMD, Palma-Rigo K, NguyenHuu TP, Morris BJ, et al. A novel interaction between sympathetic overactivity and aberrant regulation of renin by miR-181a in BPH/ 2J genetically hypertensive mice. Hypertension. 2013;62:775-81.

9. Jackson KL, Head GA, Gueguen C, Stevenson ER, Lim K, Marques FZ. Mechanisms responsible for genetic hypertension in Schlager BPH/2 mice. Front Physiol 2019;10:1311.

10. Sharma NM, Nandi SS, Zheng H, Mishra PK, Patel KP. A novel role for miR-133a in centrally mediated activation of the reninangiotensin system in congestive heart failure. Am J Physiol Heart Circ Physiol. 2017;312:H968-H979.

11. Dorr O, Liebetrau C, Mollmann H, Gaede L, Troidl C, Lankes S, et al. Effect of renal sympathetic denervation on specific microRNAs as an indicator of reverse remodeling processes in hypertensive heart disease. J Clin Hypertens (Greenwich). 2016;18:497-502. 